\title{
Evolution in random environment and structural instability
}

Sergey VAKULENKO and Dima GRIGORIEV

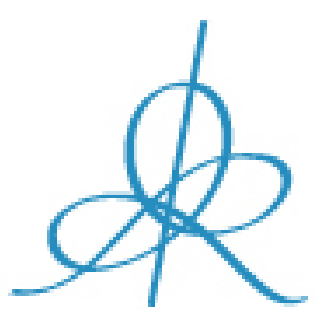

Institut des Hautes Études Scientifiques

35 , route de Chartres

91440 - Bures-sur-Yvette (France)

Avril 2006

IHES/M/06/20 


\title{
Evolution in Random Environment and Structural Instability
}

\author{
S. Vakulenko ${ }^{1}, \quad$ D. Grigoriev ${ }^{2}$ \\ ${ }^{1}$ Institute of Mechanical Engineering Problems, S. Petersburg, Russia \\ 2 IRMAR, Rennes University
}

\begin{abstract}
We consider stability and evolution of complex biological systems in particular, genetic networks. We focus our attention on supporting of homeostasis in these systems with respect to fluctuations of an external medium (the problem is posed by M. Gromov, A.Carbone [32]). Using a measure of stochastic stability we show that a generic system with fixed parameters is unstable, i.e., the probability to support homeostasis converges to zero as time $T \rightarrow \infty$. However, if we consider a population of unstable systems, which are capable to evolve (change their parameters), then such a population can be stable as $T \rightarrow \infty$. This means that the probability to survive may be non-zero as $T \rightarrow \infty$.

Evolution algorithms, that provide stability of populations, are not trivial. We show that the mathematical results on evolution algorithms are consistent with experimental data on genetic evolution.
\end{abstract}

\section{Introduction}

\subsection{Structural stability}

R. Thom, in the book [1], has proposed the concept of structural stability to describe mathematically complex structures emerging in biology and other applications. This approach has been developed and successfully applied by many authors ( catastrophe theory, see [2] for a overview).

However, this concept also leads to some difficulties [4]. For example, theory of dynamical systems shows that structurally stable systems are not dense (S. Smale, [3, 4, 5]). There are examples of chaotic structurally stable dynamical behaviour (hyperbolic dynamics, see [3, 5]), but a typical system issued from applications and exhibiting a complicated large time behaviour, is not structurally stable. It is difficult to find systems defined by polynomial differential equations satisfying the Smale criterium of structural stability. We have a similar problem 
with so-called stable maps: generically, they are not dense [6]. On the other hand, computer investigations of models of ecological systems show that, in many cases, they are not stable [8]. A great discussion on this problem can be found in [7], chapter 21, where the final conclusion is that the general notion of stability does not make a great sense for ecological systems. The stability strongly depends on the type of perturbations. We know, for example, that human interventions often destroy ecological systems.

Our start point is a remark from [32], where M. Gromov and A. Carbone formulated the following problem: "Homeostasis of an individual cell cannot be stable for a long time as it would be destroyed by random fluctuations within and without cell. There is no adequate mathematical formalism to express the intuitively clear idea of replicative stability of dynamical systems" ([32], p.40).

This assertion formulates two hypothesis. First, that functioning of biological systems are unstable under random perturbations. Second, these systems can be stabilized by replication (evolution).

The goal of this paper is to formulate mathematically and prove these hypothesis for some classes of systems important in biology, chemistry and other applications. We introduce a measure of homeostasis stability under random perturbations. After, we show that, in a sense, almost all individual systems with fixed parameters are actually unstable for large times $T$, however, populations of evolving systems with changing (from time to time) parameters can be stable even as $T \rightarrow \infty$. Our approach to this homeostasis problem uses probabilistic methods, some ideas on structural stability (in particular, R. Thom's results) and the algorithms theory. We demonstrate that this approach explains some fundamental properties of biological evolution (see Section 3 and Conclusion).

\subsection{Outline of the approach}

Recall that homeostasis means supporting of life functions of the cell (or another biological system). It is well known that biological molecules and chemical mechanisms in the cell are very fragile. Thus, in order to support their functioning, some main characteristics of the cell (temperature, pressure, $\mathrm{pH}$, reagent concentrations) must be within some narrow domain [17] independently of external medium oscillations. For example, the temperature of a human body must lie within $35-42 C^{0}$. Sharp changes in the external medium can lead to "ecological catastrophes". Therefore, it is important for investigation of biological, ecological (and economical) 
systems to take into account different fluctuations because these systems can survive only when their states stay within some bounded domains (we denote these domains by П).

Basing on these ideas, we study some models important for biological, ecological and other applications. These models contain a dynamical component and a stochastical part describing a random environment. For such models a natural measure of the stochastical stability can be introduced. This measure is a probability $P_{T}(\Pi)$ that for $t \in[0, T]$ the system state (that can evolve in time) stays in the domain $\Pi$. This measure is well known and studied [33]. For brevity, if the system state stays within $\Pi$ for $t \in[0, T]$, we say that our system survives on $[0, T]$. For a system population ( a set of the systems) we shall say that this population survives if at least one of these systems survives.

Besides this stability measure, in this paper the idea of a "generic" system plays the key role. The systems under consideration can depend on different parameters $\mathcal{P}$. Following standard ideas [10], we say that a property holds for a generic system if this property holds for an open dense set in the space of possible values of the parameters $\mathcal{P}$. In other words, this property holds for almost all systems (see [11], where one can find details and an interesting discussion of this topic).

For the models under consideration we show their instability if their parameters $\mathcal{P}$ are fixed. More precisely, we show that the survival probability $P_{T}(\Pi) \rightarrow 0$ as $T \rightarrow \infty$ for a generic system. For some important particular class of the systems (genetic circuits), this property holds for any circuits and the probability $P_{T}(\Pi)$ can be estimated.

The same result on stochastic instability holds for a finite population of systems $S_{i}$ with parameters $\mathcal{P}_{i}$, which, in general, can be distinct but fixed in time. Then again we have $P_{T}(\Pi) \rightarrow 0$ as $T \rightarrow \infty$.

The main idea is that a system evolution can stabilize system populations. If we consider a set of unstable systems with parameters $\mathcal{P}_{i}(t)$, which can change from time to time, then the limit of the survival probability $P_{T}(\Pi)$ as $T \rightarrow \infty$ may be different from 0 . Briefly, a fixed system is almost always unstable but a chain of evolving systems may be, in a sense, stable.

In the next part of the paper we investigate stable evolution algorithms such that $\lim P_{T}(\Pi)>$ 0 as $T \rightarrow \infty$, when a chain of evolving unstable systems has non-zero chances to survive for large times. In this part, our goal is to explain, with the help of this stability approach, the main property of evolution (why a system must make its copies and the mutation probability is 
small, why the genetic code size must increase during the evolution process (in average), why the evolution tree must be, in a sense, large etc).

To proceed it, we introduce a concept of a priori computational complexity of evolution problems. It allows us to apply some ideas and notions from complexity theory [43, 41, 44]. Indeed, it seems that many evolution problems are, in a certain sense, "complex". Roughly speaking, since "almost all" systems are unstable, to construct a stable system is a "complex" problem. In fact, even for simplified models the evolution algorithm must resolve NP-hard problems (about NP-hardness see books [43, 44]). We formulate some such problems.

We also find some interesting properties of evolution algorithms for genetic networks. This question is connected with the graph evolution theory pioneered by Erdos and Rényi [12] since circuits can be associated, in a natural way, with directed graphs. We show that the ErdosRényi evolution is unstable.

This approach allows us to formulate mathematically some key biological questions. For example, a very intriguing question, is whether evolution advanced step by step, or there were great jumps. This problem has been considered in many books and papers, see [18] and references in it. The answer, by our opinion, is connected with the following difficult mathematical problem: for some NP-hard problems, whether there exists greedy algorithms, which solve these problems for a certain subclass of instances.

\section{Models}

\subsection{Stochastic differential equations.}

A sufficiently general model for a dynamical evolution in random environment can be defined by

$$
\frac{d x_{i}}{d t}=F\left(x_{1}, \ldots, x_{n}, \xi_{1}, \ldots, \xi_{m}\right),
$$

where $x=\left(x_{1}, x_{2}, \ldots, x_{n}\right)$ are unknown functions of time (giving system states), $\xi_{i}$ are some random processes with piecewise continuous trajectories describing fluctuations of an external medium or an internal noise.

To simplify eq. (2.1), we can linearize at $\xi_{i}$ that gives us a model defined by the stochastic differential equations

$$
d x(t)=f(x) d t+\sum_{j=1}^{l} g_{j}(x) d w_{j}
$$


where a function $f(x)$ defines a non-random contribution into dynamics, $g_{j}(x)$ are functions and $w_{j}$ can be independent white noises (standard Wiener processes). Eq. (2.2) presents the classical physical model [15].

These models are too abstract and to obtain results that can be compared with experimental data, we describe below an important particular model describing gene or neuron interactions.

\subsection{Network models.}

In last decade, large attention is given to problems of global organization, stability and evolution of complex networks such as protein and gene networks, networks of metabolic reactions, neural and economical circuits, Internet etc. (see [29, 30, 31], for a overview [14]).

The simplest mathematical model of such network is a (directed) graph. For example, for a gene network we can associate with this network a graph where a node describes a gene, the $i$-th node is connected with the $j$-th one if the corresponding genes interact. The evolution of such graphs can be considered as an algorithm adding or removing edges and nodes. Stability can be examined in different contexts. For example, we can examine how much edges (or nodes) must be eliminated (in average) in order to destroy connectivity of the graph. In biological applications, such an elimination may simulate mutations.

The first theory of graph evolution was proposed by Erdos and Rényi $[12,14]$. They supposed that, at time moments $0,1,2, \ldots$, one adds to graph a new edge with probability $p$. This theory leads to a Gaussian distribution of $\bar{C}(k)$ of the valency of a node. Recall that the valency of a node is the number of the nodes adjacent to this node. The quantity $\bar{C}(k)$ is the probability that a node has $k$ adjacent nodes [14]. Recently it was investigated that real networks has another structure, namely, so-called scale-free structure. Here $\bar{C}(k) \approx$ const $k^{-\gamma}$, where the exponent $\gamma$ lies usually within $(2,3)$. Such networks have few number of nodes with a great valency, whereas the most of the nodes have a small valency.

Other interesting properties of graphs associated with actual biological, informational and economical systems can be described as follows. The graph diameter is restricted (the diameter is the maximal length of the shortest path connecting two nodes). The diameter defines the speed of dynamical processes in the circuit, thus a small diameter is useful to survive in the random environment. Moreover, studying of biological circuits showed that the averaged valency $\langle C\rangle$ has increased during evolution. Here $\langle C\rangle$ can be computed by $\bar{C}(k):\langle C\rangle=\sum_{k} k \bar{C}(k)$. Another property found experimentally is that more connected proteins are more important 
for organisms: letality correlates with valency.

Stability of the free-scale structures is high with respect to a random attack when one chooses nodes to eliminate randomly. However, this stability is weak with respect to a terroristic attack (when one eliminates the most connected nodes).

The first evolution algorithm leading to the scale-free organization was proposed by AlbertBarabasi [14]. This algorithm uses the idea of so-called preferential attachment: the probability that a new edge is incident to the $i$ - th node is proportional to the valency of this node.

In this paper our attention is focused on stochastic stability of the networks with respect to fluctuations describing an internal noise and oscillations of an environment. To achieve this goal we have to extend simple graph models. In fact, metabolic reaction networks or gene networks cannot be described completely as simple graphs. They define some complex dynamical system, where a scheme of interaction of substrats,ferments or genes can be associated with a graph. A part of the substrats enters this system from an external medium (input) and another part can be considered as an output (products). It is well known that these systems succesfully support an output independent of fluctuating input [19, 17].

It is difficult to describe in details global dynamical systems for metabolic reactions or gene interactions. Genetic circuit models were proposed ( $[20,22,24,26]$ among many others, see [23] for a overview) to take into account theoretical ideas and experimental information on gene interactions. Model [22] uses Boolean algebra (so-called Boolean switch network). Models $[24,26]$ can be considered as a generalization of the famous Hopfield model of attractor neural network [16]. To simplify situation, we focus our attention on this particular model, which is based on two main ideas. The first one is to choose the gene concentrations as state variables for the description of gene regulation. The second one is to take into account a pair interaction betwenn genes, to describe activation or depression of one gene by another. We consider a simplified variant of the equations [24, 25], where diffusion is removed, namely

$$
\frac{d u_{i}}{d t}=R_{i} \sigma\left(\sum_{j=1}^{m} K_{i j} u_{j}+\theta_{i}-\xi_{i}(t)\right)-\lambda_{i} u_{i},
$$

where $m$ is the number of genes included in the circuit, $u_{i}(t)$ the concentration of the $i$-th gene, $\lambda_{i}$ the gene decay rates, the parameters $\theta_{i}$ are activation thresholds, $\xi_{i}(t)$ describe random fluctuations, and $\sigma$ is so-called sigmoidal function (see below). We assume that the $\xi_{i}$ are random processes with piecewise continuous trajectories. 
The real number $K_{i j}$ measures the influence of the $j$-th gene on the $i$-th one. The initial data are

$$
u_{i}(0) \equiv S_{i}
$$

where $S_{i}$ are random numbers. The function $\sigma$ is a strictly monotone increasing function satisfying

$$
\lim _{z \rightarrow-\infty} \sigma(z)=0, \quad \lim _{z \rightarrow \infty} \sigma(z)=1 .
$$

The well known example is $\sigma(z)=\frac{1+\tanh (z)}{2}$. Another important example of $\sigma$ is given by so-called Michaelis- Menten function. This function $\sigma$ equals $x /(K+x)$, where $K$ is a positive constant, for positive $x$ and equals 0 for $x \leq 0$.

Model (2.3) takes into account only two fundamental processes: a) the decay (degradation) of gene products (the term $-\lambda_{i} u_{i}$ ); and b) gene regulation and synthesis.

Another possible model is a dynamical system with discrete time, for example, defined by the following iterative process

$$
\begin{gathered}
u_{i}^{t+1}=r_{i} \sigma\left(\sum_{j=1}^{m} K_{i j} u_{j}^{t}+\theta_{i}-\xi_{i}^{t}\right)-\lambda_{i} u_{i}, \\
u_{i}^{0} \equiv S_{i},
\end{gathered}
$$

where $t=0,1,2, \ldots, T, T$ is an integer, $\xi_{i}^{t}$ are random functions of dicrete time $t$. Numerical procedures solving (2.3) lead to models similar to (2.8).

In this paper we focus our attention to model (2.6) although many results can be extended to case (2.3) (however here the proofs are more complicated). Systems (2.5) without stochastic effects have been studied analytically in [45]. It was shown that they generate any spatiotemporal patterns.

\section{Main results}

Let us formulate now main mathematical results. Their biological interpretation and comparison with experimental data is given in Conclusion.

\subsection{Results for circuits}

We consider the question on the stochastic stability of genetic circuits (2.5). 
A For genetic circuits we obtain that the more is the valency of a node the stabler is the circuit with respect to perturbations in this node. We also prove that the survival probability of each circuit of a fixed structure tends to zero as $T \rightarrow \infty$. Therefore, "homeostasis" generated by a fixed circuit will be broken as time tends to infinity.

B We show that although a fixed isolated circuit is always stochastically unstable (see previous item), a chain of circuits could be stable. In this chain, each circuit is obtained from the previous one by some algorithm modifying the circuit parameter (replication algorithm). Roughly speaking, to survive, it is necessary to evolve, but in a special way. We investigate the evolution algorithms leading to a stable ("eternal") evolution when $\lim _{T \rightarrow \infty} P_{T}(\Pi)>0$. We show that the mean valency must increase during such stable evolution. Moreover, we prove that the Erdos- Rényi algorithm of graph evolution is unstable, i.e., $\lim _{T \rightarrow \infty} P_{T}=0$. Moreover, we find a connection between evolution problems and some NP-complete problems for graphs.

\subsection{Results for stochastical equations}

C We show that "generic" model (2.2) with fixed and smooth parameters $g_{j}$ are stochastically unstable in a sense that $P_{T} \rightarrow 0$ for $T \rightarrow \infty$. For model (2.1) with a polynomial nonlinearity $F$ we find a connection between the stochastic instability and problems of real algebraical geometry. In this case the evolution algorithm resolves certain problems of real algebraic geometry. The known results $[36,35]$ allow us to estimate the running time of evolution algorithms. This estimate is an upper estimate (possibly, there are more effective algorithms).

\subsection{General properties of stable evolution algorithms}

Under some natural assumptions (the parameter evolution is a Markov process, parameters lie in a discrete set $\mathcal{D}$ and others) ) one can describe some general properties of stable evolution processes. The most interesting property is the following. The evolution, to be stable, should be close to a replication, and the size $|D|$ of the set $D$ ("genetic code" size) increases during evolution.

The last property shows that it is natural to consider evolution as a Markov process with a countable set of states, for instance, as a branching process associated with a tree. Using ideas of the algorithm theory, we also give some estimates of this tree size. The tree must be big (if $P \neq N P)$.

\subsection{Outline of the proofs}

The proof of $\mathbf{A}, \mathbf{B}$ and $\mathbf{D}$ is quite straightforward and uses some elementary probabilistic 
and algorithmic arguments. The study of (2.1), (2.2) (result $\mathbf{C}$ ) is based on the known results for stochastical problems [33] and also use the known result of C. Lobry [9] on so-called polydynamical systems. It is interesting to note that this result, in turn, is based on the Thom transversality theorem [10].

\subsection{Organization of the paper}

We state the results $\mathbf{A}$ in Section 4. Section 5 concerns the stability of evolution algorithms. For gene circuits, we deduce some estimates for survival probabilities $P_{T}$. Basing on these estimates, we investigate stochastic stability of Erdos-Rényi algorithm. Here we also find a connection of evolution problems with some NP-complete problems for graphs. In Section 6 we consider stochastic differential equations (2.1) and (2.2) and show their stochastic instability in generic situation. In Section 7 we investigate general properties of evolution algorithms. Thanks to the concept of a priori computational complexity we obtain that the evolution tree must be unboundedly increasing as $T \rightarrow \infty$.

In conclusion we compare main results with biological experimental data. We show a good accordance in many key points.

\section{Stochastic Stability for Circuits}

The important meaning has the problem of stability of networks under random perturbations of different parameters. This problem attracts a great attention of biologists (see [29, 30, 31]). We obtain some estimates on stability of (2.8) under noise leading to important biological consequences.

Consider problem (2.8) with $\lambda_{i}=0$ :

$$
\begin{gathered}
u_{i}^{t+1}=\sigma\left(\sum_{j=1}^{m} K_{i j} u_{j}^{t}+\theta_{i}-\xi_{i}^{t}\right), \\
u_{i}^{0}=S_{i},
\end{gathered}
$$

where $\xi_{i}^{t}$ are some random processes with the discrete time, $S_{i}$ are random numbers. We assume that $\xi_{i}^{t}$ are independent for different $i$. Different choices of the distributions for $\xi_{i}^{t}$ and $S_{i}$ may correspond to different "ecological conditions". The processes $\xi_{i}^{t}$ simulate an internal noise in the system whereas $S_{i}$ may simulate fluctuations of inputs. For example, if system (4.1) describes a metabolic network, fluctuations of $S_{i}$ are oscillations of nutrients and $\xi_{i}^{t}$ can be 
associated with oscillations of temperature, pressure or $\mathrm{pH}$. Strong jumps of the value $\xi_{i}^{t}$ for the $i$-th node can be interpretated as "mutations" removing this node from a working circuit.

Let us introduce functions $\Psi_{i}$ by

$$
\operatorname{Prob}\left\{\xi_{i}^{t}<a \text { for all } t \in\left[T_{1}, T_{2}\right]\right\}=\Psi_{i}\left(a, T_{1}, T_{2}\right)
$$

The following assumption plays an important role in what follows. Suppose

$$
\Psi_{i}\left(a, T_{1}, T_{2}\right)>0, \quad \Psi_{i}\left(a, T_{1}, T_{2}\right) \rightarrow 0 \text { as } T_{2} \rightarrow \infty
$$

for any $T_{1}$ and $T_{2}$ such that $T_{2}>T_{1}$. Roughly speaking, this means that $\xi_{k}^{t}$ can take any large values with non-zero probabilities.

We say that a system (a circuit (4.1))"survives" (supports homeostasis) if the concentrations $u_{i}$ lie inside a closed domain $\Pi$ in the $u$-phase space. Notice that our conditions on $\sigma$ (see (2.5)) entail $u_{i}^{t} \in(0,1), \quad t>0$.

It is natural therefore to suppose that $\Pi$ is contained inside the cube $[0,1]^{m}$. As a measure of the stochastic stability of the circuit homeostasis, we consider the probability

$$
P\left(\mathcal{P}, \Pi, T_{1}, T_{2}\right)=\operatorname{Prob}\left\{\mathbf{u}^{t} \in \Pi \text { for each } t \in\left[T_{1}, T_{2}\right]\right\},
$$

where $\mathbf{u}^{t}=\left(u_{1}^{t}, \ldots, u_{m}^{t}\right)$. This probability depends on the circuit parameters $\mathcal{P}$ and the homeostasis domain $\Pi$. We shall name it the survival probability on the time interval $\left[T_{1}, T_{2}\right]$ and denote by $P\left(T_{1}, T_{2}\right)$ omitting the dependence on the parameters $\mathcal{P}, \Pi$.

One can consider a more realistic and complex case when we deal with a family of different perturbations depending on a random parameter $\omega \in \Omega_{0}$ and for each $\omega$ we have the corresponding box $\Pi_{\omega}$. Such situation is typical in biology: for example, if the environment contains a lot of nutrient, genes connected with production of this nutrient may be blocked [17, 19]. However, to simplify estimates, we shall not consider this case here.

We estimate the stability via the following parameters: the valency, $\left|K_{*}\right|$, the maximum $b$ of $\left|\theta_{i}\right|$ and some parameter $N_{\text {key }}$ that will be introduced below. It is important to take into account the valency since it is well known that biological circuits are far from being completely connected: for each fixed node $i$ we have a valency $V_{i}<m$ : only $V_{i}$ among the entries $K_{i j}$ are not equal zero. In applications, typically, $V_{i}<<m[14]$.

To define $N_{k e y}$, let us observe first that

$$
\inf _{\mathbf{u} \in \Pi} u_{i}=W_{i} \geq 0
$$


Denote $U_{i}=\sigma^{-1}\left(W_{i}\right)$. Some $W_{i}$ and $U_{i}$ could be positive. The corresponding indices $i_{1}, \ldots, i_{s} \in$ $[1, \ldots, m]$ will be named key indices and the corresponding genes will be named the key ones. In fact, assumption $W_{i}>0$ means that the organism cannot survive if the concentration of $i$ -th gene is small enough. The number of the key genes is denoted by $N_{k e y}$. We denote by $I$ the set of the key indices corresponding to the key genes.

Let us fix some key index $i \in I$ and consider (4.1). We have the following simple inequality

$$
\sum_{j=1}^{m} K_{i j} u_{j}^{t}+\theta_{i}-\xi_{i}^{t} \leq S_{i}=V_{i} K_{*}+b-\xi_{i}
$$

Thus, if

$$
\xi_{i}^{t}>V_{i} K_{*}+b-U_{i},
$$

the concentration $u_{i}^{t+1}$ is less than the critical value $W_{i}$. Moreover, if at least one $u_{i}^{t}$ is less than $W_{i}$, the state $\mathbf{u}^{t}$ is outside of this domain $\Pi$. Hence, we have

$$
\operatorname{Prob}\left\{\mathbf{u}^{t} \in \Pi, t \in\left[T_{1}+1, T_{2}\right]\right\} \leq \prod_{i \in I} \Psi_{i}\left(V_{i} K_{*}+b-U_{i}, T_{1}, T_{2}-1\right) .
$$

Therefore, we have proved

Proposition 4.1. The survival probability satisfies

$$
P\left(T_{1}, T_{2}\right) \leq \prod_{i \in I} \Psi_{i}\left(V_{i} K_{*}+b-U_{i}, T_{1}-1, T_{2}-1\right)=R\left(T_{1}, T_{2}\right) .
$$

This estimate implies the following consequences. Notice that the function $R$ is a monotone increasing function of the valencies $V_{i}$. Moreover, we notice that all circuits are stochastically unstable as the time $T$ goes to infinity. In fact, assumption (4.4) and estimate (4.9) imply

$$
P(0, T) \rightarrow 0 \quad \text { as } T \rightarrow \infty .
$$

Then there arises a natural question: how to stabilize the circuits. We shall consider this problem in the coming section.

\section{$5 \quad$ Evolution Stability for Circuits}

\subsection{General approach}


In this section we show that a time evolution of the circuit parameters $\mathcal{P}$ can transform stochastically unstable systems to the stable ones. The key question is about evolution properties providing the stability.

We consider circuits (4.1) under the assumptions of the previous section. We also suppose that $\xi_{i}^{t}$ are identical independent random processes, which are homogeneous in time. More precisely, let us assume

$$
\Psi_{i}\left(a, T_{1}, T_{2}\right)=\Psi_{i}\left(a, 0, T_{2}-T_{1}\right) .
$$

Consider possible schemes of circuit evolution. They can be described as follows.

Each $T_{r}$ time steps we change the circuit parameters $\mathcal{P}$ following some rule. For example, each $T_{L}$ time steps we can add to the network a new edge, and each $T_{n}$ steps, we include a new node (gene). Here $T_{n}$ and $T_{L}$ are some positive integers. We can also use more sophisticated schemes. For example, one can add new nodes with many edges. In the case of graphs, different schemes of graph evolution were studied in numerous works, see the overview [14].

Let us calculate the survival probability. Let $P_{n}=P\left(\mathcal{P}_{n},\left[n T_{r}, n T_{r}+T_{r}\right]\right)$ be the probability to survive within the time interval $\left[n T_{r},(n+1) T_{r}\right]$. Here $\mathcal{P}_{n}$ are the circuit parameters in this time interval.

The probability to survive on the interval $(0, \infty)$ is then the infinite product

$$
P(0, \infty)=P_{1} P_{2} P_{3} \ldots=\prod_{n \in \mathbf{N}} P_{n} .
$$

Consequently, the quantity $P(0, \infty)$ is non-zero if the series $\log P_{1}+\log P_{2}+\ldots+\log P_{n}+\ldots$ converges. We have obtained thus the following assertion.

Proposition 5.1. If the series

$$
\sum_{n=1}^{\infty} \log P\left(\mathcal{P}_{n},\left[n T_{r},(n+1) T_{r}\right]\right)
$$

converges, the survival probability $P(0, T)$ remains positive as $T \rightarrow \infty$. If this series diverges to $-\infty$, the survival probability tends to zero as time tends to infinity.

Propositions 4.1 and 5.1 yield a necessary condition for stochastic stability in infinite time. Notice that it is more precisely to say about stochastic stability of the pair (circuit, evolution algorithm) rather than about stochastic stability of just the circuits.

Proposition 5.2. Denote

$$
\left.Z_{n}=\sum_{i \in I} \log \Psi_{i}\left(V_{i}^{n} K_{*}+b-U_{i}, n T_{r},(n+1) T_{r}\right)\right)
$$


where $V_{i}^{n}$ are the valencies at the $n$-th renovation step. If the series $Z_{1}+Z_{2}+\ldots+Z_{n}+\ldots$ diverges, then the survival probability $P(0, T)$ tends to zero as $T \rightarrow \infty$.

To prove it, let us notice that, due to Proposition 4.1, $-\log P\left(T_{1}, T_{2}\right)>-\sum_{i \in I} \log \Psi_{i}\left(V_{i} K_{*}+\right.$ $\left.b-U_{i}, T_{1}-1, T_{2}-1\right)$.

By these results we can analyze different evolution algorithms. Consider an example.

\section{Example: Erdos - Rényi evolution.}

Let us suppose that only a part of all the nodes are key ones. The simplest case is $|I|=1$ ( $I$ consists of a single node).

Let us consider a simple case when the number of the key nodes is constant during evolution. Each new node is therefore not a key one. We can compare two case: Erdos -Rényi evolution model, when a new node may be connected with any nodes with equal probability and the Albert-Barabasi model (remind that the latter model uses the idea of the preferential attachment: the probability that a new edge is incident to $i$-th node is proportional to the valency of this node).

We consider first the classical model of graph evolution, Erdos - Rényi evolution. We consider random graphs with $N=m$ nodes and $M$ edges, and we will study the asymptotics as $N \rightarrow \infty$. We suppose that these $M$ edges are chosen at random among the $E_{N}=\left(\begin{array}{l}N \\ 2\end{array}\right)$ possible edges so that all $E_{N}$ possible choices are equiprobable.

An evolutionary equivalent formulation is the following: let us suppose $N$ labelled points $p_{i}$ (nodes) are given. Let us choose randomly an edge among the $E_{N}$ possible edges, after this an edge among the $E_{N}-1$ remaining edges etc., and so on, in all we make $M$ choices.

With considered graphs we associate dynamical circuits (4.1) setting $K_{i j}=1$ if the $i$-th and $j$-th nodes are connected and $K_{i j}=0$ otherwise. Let us estimate the probability $P(0, M, N)$ to survive within $M$ steps for large $M, N$. Suppose the marked key node is the 1-th node. We will write, for brevity, $\Psi_{1}(a, T, T+1)=\Psi(a)$, since this function is indepedent of $T$ (see assumption (5.1)). Furthemore, let $V^{1}, V^{2}, \ldots, V^{M}$ be valencies of this key node at the first, second, ... $M$-th step of evolution, respectively. It is clear that $V^{1} \leq V^{2} \ldots \leq V^{M}$, i.e., the sequence $V^{n}$ is increasing.

Repeating the arguments of this section, we obtain, due to this mononicity, the estimate

$$
P(0, M, N) \leq \mathbf{E}_{\text {s.r.g }} \Psi\left(V^{1}\right) \Psi\left(V^{2}\right) \ldots \Psi\left(V^{m}\right) \leq \mathbf{E}_{\text {s.r.g }} \Psi\left(V^{M}\right)^{M}=\mathbf{E}_{r . g} \Psi\left(V^{M}\right)^{M},
$$

where $\mathbf{E}_{\text {s.r.g }}$ means the mathematical expectation over all sequencies of random graphs, $\mathbf{E}_{r . g}$ 
is the expectation over all random graphs with $E_{N}$ nodes and $M$ edges. Denote by $p_{k}$ the probability that such a random graph has exactly $k$ nodes adjacent to the first node. Repeating the estimates of Erdos-Rényi ([12], see as well [13]), we can deduce the estimate

$$
p_{k} \leq(k !)^{-1}\left(\frac{c M}{N}\right)^{k}
$$

Then inequality (5.4) can be rewritten as

$$
P(0, M, N) \leq \sum_{k=0}^{N-1} p_{k} \Psi(k)^{M} \leq \sum_{k=0}^{M}(k !)^{-1}\left(\frac{c M}{N}\right)^{k} \Psi(k)^{M} .
$$

Let us formulate now an assertion.

Proposition 5.2 Suppose $M \leq C_{0} N$, where $C_{0}$ is a constant. Then $P(0, M, N) \rightarrow 0$ as $N \rightarrow \infty$, i.e., the Erdos-Rényi algorithm is unstable.

To prove this assertion, let us take a small $\epsilon>0$. Suppose $M \rightarrow \infty$ as $N \rightarrow \infty$ (for bounded $M$ and $N \rightarrow \infty$, our assertion is a trivial consequence of $(5.4 \mathrm{a}))$. Notice that $\Psi(k) \leq 1$. Therefore, for any integer $n \in[0, M]$ one has

$$
P(0, M, N) \leq \sum_{k=0}^{n}(k !)^{-1}\left(c C_{0}\right)^{k} \Psi(k)^{M}+\sum_{k=n+1}^{M}(k !)^{-1}\left(c C_{0}\right)^{k} .
$$

The second sum can be made less than $\epsilon / 2$ by a choice of a large $n=n(\epsilon)$. Let us choose such $n$ and fix it. Suppose $M$ tends to $\infty$. Then the first sum converges to zero, since $\Psi(n)^{M} \rightarrow 0$ for fixed $n$. So, for large $N$ we have $P(0, M, N)<\epsilon$ and our assertion is proved.

If the key nodes at initial moment have essentially more adjacent nodes than the rest nodes, then it is clear that the Albert-Barabasi algorithm gives to the circuits essentially more chances to survive than the Erdos-Rényi one. Indeed, the preferential attachment algorithm produces a graph, where the valency of key nodes will be much more than for a graph generated by the Erdos-Rényi algorithm.

\subsection{Evolution as a computational problem. Relation to some NP-complete prob-} lems

In the previous subsection we have explained that a realistic model of circuit evolution must use a nontrivial algorithm, of Albert-Barabasi type, or even more complex. Let us consider now some restrictions to possible connections in graph $K$ taking into account a real structure of biological molecules. 
Above, analyzing different approaches, we have supposed that during evolution process any two nodes could be connected. This could give an impression that network evolution is an easy process. Actually, however, this evolution cannot be such a simple process, and we shall see it in this subsection.

Biomolecules consist of numerous polymer groups, and in a chemical reaction, they loose (or accumalate) only one such group. This explains, in particular, why enzyme reactions proceed in many steps (see [19]). We conclude therefore that if our graph describes, for example, a scheme of metabolic reactions, then it is impossible, in general, to connect two arbitrary nodes. An analogous picture can be observed for other real graphs. Consider, for instance, a graph describing coauthors. One can expect, a priori, the probability that a specialist in physics will write together with a specialist in abstract algebra is essentially less than the probability of cooperation for two physicists.

To take into account possible natural restrictions on the matrix $K$ fixed a priori, we can introduce a large graph $(V, E)$, where $V$ is a set of nodes, $E$ is a set of edges.

With each $v_{j} \in V$ we can associate a chemical reagent $u_{j}$. The entry $K_{i j}$ in (4.1) could be non-zero only if it prescribed by $E$, i.e., when $v_{i}, v_{j}$ a priori can be connected $\left(v_{i}, v_{j} \in E\right)$.

Now an evolution can be formally described as a time change of subgraphs $\left(V, D^{t}\right), D^{t} \subset E$, where $t=0,1,, 2, \ldots$ and $D^{0} \subset D^{1} \subset D^{2} \ldots$. To obtain in such a way a complicated chemical reaction transforming a substrat $s \in V$ to a product $p \in V$, we must therefore find a simple path in $(V, E)$ leading from $s$ to $p$.

It is clear as well that the length of this way may be large, but a priori restricted by a number $L_{\max }$. Otherwise, the relaxation processes will be very long and such a system could not survive.

Let us recall our main principle, namely, that the system must be stable in stochastical environment. This implies, in particular, that the system should be stable with respect to mutations or random vanishing of some substrats needed for producing the product $p$. Mutations can lead to elimination of some nodes or edges (see above beginning of Section 2).

To provide such stability, evolution should form more than one way from different nutrients to products. The more different ways we have, the stabler is our system. Thus, we obtain the following problem:

Problem 5.1 Given a graph $G=(V, E)$, collection of disjoint node pairs $\left(s_{1}, \bar{s}_{1}\right), \ldots,\left(s_{k}, \bar{s}_{k}\right)$. 
Does $G$ contain $J$ or more mutually pairwise node-disjoint simple paths connecting $s_{i}$ and $\bar{s}_{i}$ for each $i=1, \ldots, k$ ?

This problem is NP-complete (see [43]). The given nodes $s_{i}$ could correspond to nutrients (substrates), nodes $\bar{s}_{i}$ could correspond to products, the paths correspond then to some metabolic paths. Suppose that a system, defined by the graph, survives if the environment contains at least one type of nutrients $s_{i}$. The random fluctuations are eliminations of some nutrients.

There are possible different models of such fluctuations and their action on the system. We shall distinguish two cases: hard environments and soft ones.

Example. Suppose each nutrient $s_{i}$ can vanish independently with a probability $r_{i}$. The system will be destroyed if all possible nutrients are absent. Then, if $k$ paths have been found, the probability to survive (per unit time) becomes $f(k)=1-r_{1} r_{2} \ldots r_{k}$.

We say that an environment is hard, if the function $f(k)$ (the probability to survive per unit time after a solution of $k$-th problem) admits, for large $k$, the following estimate:

$$
f(k)<1-\delta k^{-\mu},
$$

for some $\mu>0$, where $\delta>0$. Otherwise, the environment is soft. For the problem 5.1 the evolution algorithm is a finding of different paths. Problem 5.1 gives rise to a natural hierarchy of the computational problems (one path is found, two paths are found, ... $k$ paths are found).

Another natural NP-hard problem related to the stability can be formulated as follows:

Problem 5.2 Given a graph $(V, E)$, positive integers $K \leq|V|$ and $B \leq|E|$, is there a subset $\tilde{E} \subset E$ with $\left|E^{\prime}\right| \leq B$ such that the graph $\left(V, E^{\prime}\right)$ is $K$-connected, i.e. cannot be disconnected by removing fewer than $K$ nodes?

This problem is simple for $K=1$ but it is NP-hard for $K \geq 2$. In the next subsection we shall see that existence of such problems yields interesting consequences for the evolution process. Notice that a number of NP -hard problems can be associated with the models from Sections 4 and 5. We do not formulate them here. An example is given in Section 7.

\section{$6 \quad$ Instability for stochastic equations}

\subsection{Smooth generic systems}


We consider here the case $l=2$ and simplifying notation, we denote $g_{1}=g, g_{2}=h$. The case $l>2$ can be considered analogously. For $l=1$ the results below are invalid.

We shall write eq. (2.2) in the following form

$$
\frac{d x(t)}{d t}=f(x)+g(x) \xi_{1}(t)+h(x) \xi_{2}(t), \quad x(0)=x^{0},
$$

where $x \in \mathbf{R}^{n}, \xi_{1}, \xi_{2}$ are random processes with continuous trajectories. Conditions on $\xi_{i}$ will be formulated below.

We associate with $(6.1)$ the so-called polydynamical system $(g, h)[9]$ generated by vector fields $g$ and $h$. An orbit of this polydynamical system can be obtained as follows: first, for $t \in\left[0, t_{1}\right]$ we move along an orbit generated by the field $g(x)$, then for $t \in\left[t_{1}, t_{2}\right]$ along an orbit generated by the field $h(x)$ etc.

Consider all orbits of this polydynamical system starting from a point $x_{0}$. Let $U$ be an open bounded neighborhood of this point in $\mathbf{R}^{n}$.

Lemma 6.1. Suppose $s \geq n^{2}+n$. The following property of the polydynamical system $(g, h)$ associated with (6.1) is "generic" for smooth fields $g$ and h, i.e., it holds for an open dense set of $C^{s}$ - fields $g, h$ (where the set of all vector fields is endowed with the standard Whitney topology, see [10]).

For all $x_{1} \in U$ there exists an orbit of the polydynamical system connecting $x_{0}$ and $x_{1}$.

This assertion actually is a particular case of the known theorem obtained by C. Lobry [9] for polydynamical systems on manifolds by R. Thom's theorem on transversality.

Using this result we prove, under some conditions, that a 'generic" system (6.1) is stochastically unstable. Suppose the set $\Pi$ that defines stochastic stability enjoys the following property:

$$
\operatorname{diam} \Pi<r, \quad r>0 .
$$

This yields that the complementary set $\mathbf{R}^{n}-\Pi$ contains a ball $B$ of a diameter $d>0$.

Suppose the processes $\xi_{i}$ satisfy the following condition. Let us introduce the distance between trajectories defined on $\left[0, t_{0}\right]$ by the $L_{p^{-}}$norm

$$
\operatorname{dist}_{p}\left(\xi(\cdot), \tilde{\xi}(\cdot), t_{0}\right)=\left(\int_{0}^{t}|\xi(s)-\tilde{\xi}(s)|^{p} d s\right)^{1 / p}, \quad p \geq 1 .
$$

Denote by $\mathcal{U}_{\delta, p, \xi}$ the tubular neighborhood of a trajectory $\xi(t), t \in\left[0, t_{0}\right]$ :

$$
\mathcal{U}_{\delta, \xi}=\left\{\eta(t): t \in\left[0, t_{0}\right], \quad \operatorname{dist}_{p}\left(\eta, \xi, t_{0}\right)<\delta\right\}
$$


Let us formulate now an important assumption on the random processes $\xi(t)$.

Assumption 6.1 Suppose for each pair of piecewise constant trajectories $\eta_{1}(t), \eta_{2}(t)$, where $t \in\left[0, T_{0}\right]$, and for any $\delta, T_{0}>0$ there holds

$$
\operatorname{Prob}\left\{\left(\xi_{1}(t), \xi_{2}(t)\right) \in \mathcal{U}_{\delta, \eta_{1}} \times \mathcal{U}_{\delta, \eta_{2}} \quad t \in\left[0, T_{0}\right]\right\}>0
$$

Using the known results [34] one can show that Assumption 6.1 holds for large classes of Markov random processes, for example, when $\xi_{i}$ are independent brownian motions. To show this last fact, we can approximate $\xi_{i}$ in the supremum norm by step functions. For each $i$ and each step the neighborhoods $\mathcal{U}_{\delta, \eta_{1}}$ can be replaced by more narrow neighborhoods $\mathcal{V}_{k}=$ $\left\{x(t), t \in[t+k h, t+(k+1) h], \quad\left|x-a_{k}\right|<\kappa\right\}$, where $a_{k}$ are constants, $k=1,2, . . N, h N=T$, $\kappa=\kappa(\delta)$ is a small positive number. The probability that $\xi_{i}(t)$ remain in all the $\mathcal{V}_{k}$ is positive (this follows from [34], Chapter VI).

\section{Proposition 6.2}

Suppose $g, h \in C^{r}, r>1$. Under Assumptions 6.1, and (6.2) generic system (6.1) is unstable, i.e., the survival probability $P_{T}(\Pi)$ converges to 0 as $T \rightarrow \infty$ uniformly in initial data $x^{0} \in \Pi$.

\section{Proof.}

Step 1 By Lemma 6.1 we can find $\xi_{1}(t), \xi_{2}(t), t \in\left[0, T_{0}\right]$ such that a trajectory $x(t)$ generated by the system

$$
\frac{d x(t)}{d t}=g(x) \xi_{1}(t)+h(x) \xi_{2}(t)
$$

goes from any start point $x_{0}$ and enters $B_{d / 2}$, where $B_{d}$ is an open ball contained in the complement $\mathbf{R}^{n}-\Pi$ of $\Pi$.

Let $a$ be a large positive number. Then the functions $\tilde{\xi}_{k}=a \xi_{k}(a t)$ on $\left[0, a^{-1} T_{0}\right]$ also generate a trajectory $\tilde{x}(t)$ of (6.1a) that attains the ball $B_{d / 2}$ within the time interval $\left[0, a^{-1} T_{0}\right]$. Let us consider now the differential equation

$$
\frac{d x}{d t}=f(x)+g(x) \tilde{\xi}_{1}(t)+h(x) \tilde{\xi}_{2}(t), \quad t \in\left[0, a^{-1} T_{0}\right]
$$

and the difference $w=\tilde{x}(t)-x(t)$. For this difference we have

$$
\frac{d w}{d t}=f(x(t))+\tilde{\xi}_{1}(t) G\left(\bar{x}_{1}(t) w+\tilde{\xi}_{2}(t) H\left(\bar{x}_{2}(t)\right) w\right.
$$


where $G(x), H(x)$ are linear operators with uniformly bounded norms $\|G\|,\|H\|<C_{0}$ and a positive constant $C_{0}$ is independent of $a$. Here $\bar{x}_{j}(t)$ are points within the interval $[x(t), \tilde{x}(t)]$. We have $w(0)=0$ (the starting points for both trajectories $x$ and $\tilde{x}$ are the same). Thus, since $|f|<C_{1}$, we obtain

$$
|w(t)| \leq \int_{0}^{t}\left(C_{1}+C_{0} a|w(\tau)|\right) d \tau
$$

Using the Gronwall inequality we find

$$
|w(t)|<C_{1} t \exp \left(C_{0} a t\right)
$$

where $C_{i}$ are uniform in $a$ as $a \rightarrow \infty$. Thus, if $a$ is large enough $w\left(a^{-1} T_{0}\right)$ is less than $d / 4$. . This entails that the trajectory $x(t)$ enters the ball $B_{d}$ at the time moment $a^{-1} T_{0}$.

Step 2 The probability of realization of the single trajectory $\tilde{\xi}_{1}(t), \tilde{\xi}_{2}(t)$ may be zero, however, repeating similar estimates, one can show that all the trajectories from the $\operatorname{set} \mathcal{U}_{\delta, \xi_{1}} \times \mathcal{U}_{\delta, \xi_{2}}$ attain $B_{d}$ if $\delta$ is small.

In fact, let us consider equation (6.5) and the same equation with other $\xi_{i}=\bar{\xi}_{i} \in \mathcal{U}\left(\tilde{\xi}_{1}, \tilde{\xi}_{2}, \delta\right)$

$$
\frac{d \bar{x}}{d t}=f(\bar{x})+g(\bar{x}) \bar{\xi}_{1}(t)+h(x) \bar{\xi}_{2}(t), \quad t \in\left[0, a^{-1} T_{0}\right]
$$

where $\delta$ is a small positive constant. Denote $v=x-\bar{x}$. Proceeding as above (Step 1 ) for $w$, we obtain a differential equation for this difference $v$ and, by integrating, we arrive at the integral inequality

$$
|v(t)| \leq \int_{0}^{t}\left(C_{2} \rho(\tau)+C_{3} a|w(\tau)|\right) d \tau
$$

where $\rho(t)=\left|\bar{\xi}_{1}(t)-\tilde{\xi}_{1}(t)\right|+\left|\bar{\xi}_{1}(t)-\tilde{\xi}_{1}(t)\right|$. By the definition of the neighborhood $\mathcal{U}$, the integral of the function $\rho$ over $\left[0, a^{-1} T_{0}\right]$ is less than $\delta$. Therefore, we can again use the Gronwall inequality that gives, by $(6.7 \mathrm{~b}),\left|v\left(a^{-1} T_{0}\right)\right|<C_{4} \delta<d / 8$ for sufficiently small $\delta$.

Therefore, the probability to enter the ball $B_{d}$ within the time interval $\left[0, T_{0}\right]$ is positive. This implies that $P_{T} \rightarrow 0$ as $T \rightarrow \infty$. Indeed, due to assumption 6.2 , we can suppose that $\Pi$ is closed and bounded, therefore, it is a compact. The estimate $P_{T_{0}}\left(x_{0}\right)<1-\delta_{1}$, where $\delta_{1}>0$, is uniform in $x_{0} \in \Pi$, where $x_{0}$ is a starting point for (6.1). Let us estimate $P_{2 T_{0}}$. This probability is less than $\left(\max P_{T_{0}}\right)^{2}<\left(1-\delta_{1}\right)^{2}$. Repeating this by induction, $P_{n T_{0}} \rightarrow 0$ as $n \rightarrow \infty$ and the proposition is proved.

Remark. This proposition can be considered as a mathematical formulation of the assertion of M. Gromov and A. Carbone [32] on the homeostasis instability (cf. above the introduction). 
"Almost all" systems (2.2) are unstable, i.e., they are not possible to support homeostasis eternelly. Similar results hold for equations (2.8).

\subsection{Polynomial dynamical systems}

The polynomial and rational dynamical systems are more natural for biological applications. It is difficult, however, to demonstrate an analogue of Prop. 6.1 for "generic" polynomial dynamical systems. Moreover, we have different notions of genericity (see a discussion in [11]). For our goals will be more useful the concept of the metrical genericity, when "almost all" means " all besides a set of zero measure".

To overcome these difficulties, we consider admissible domains $\Pi$, which, in a sense, are narrow. From biological point of view, it can be explained by fragility of biological systems. We suppose that there always exists a direction such that acting in this direction can destroy our system. To formalize this idea, we introduce the following class of domains $\Pi$.

Definition. We say that a set $\Pi \subset \mathbf{R}^{n}$ is $\delta$-narrow at the point $x_{0}$, where $\delta>0$, if there exists a unit vector $e$ such that the ray $x_{1}=x_{0}+\tau e, \tau>\delta$, lies outside $\Pi$.

The supremum over all the points $x_{0}$ of the infimum of $\delta$ satisfying this definition can be named the width of the set $\Pi$. The width determines the maximal radius of inscribed balls.

The $\delta$-narrow at $x_{0}$ set can be large in some directions, but it should be sufficiently narrow at least in one direction defined by the vector $e$.

If $\Pi$ is $\delta$-narrow at some $x_{0}$ with a $\delta$ small enough, then analysis of stochastical stability reduces to some complicated polynomial equations. We are going to use the following known results of geometric control theory.

Lemma 6.3 (Kalman criterium of controllability).

Consider the linear system

$$
\frac{d x}{d t}=A x+B \xi(t), \quad x(0)=0
$$

where $x \in \mathbf{R}^{n}, A$ is a $n \times n$ matrix, $B$ is a vector $\in \mathbf{R}^{n}$ and $\xi(t), t \in[0, T]$ is a control. Then the system (6.10) is controllable, i.e., for each $x_{1}$ there exists a $\xi(\cdot)$ such that the corresponding trajectory of (6.10) attains $x_{1}$ if and only if the following condition holds:

$$
\operatorname{dim} \operatorname{Span}\left\{B, A B, A^{2} B, \ldots, A^{n-1} B\right\}=n
$$


Consider now a system (2.1) with a polynomial right hand side $F$. To simplify situation we suppose that $m=1$, i.e., we have only one fluctuating parameter $\xi_{1}=\xi$. We investigate a stability at an equalibria of a non-perturbed system, i.e., we suppose that for $\xi=0$ there exists a point $x_{0}$ such that $F\left(x_{0}, 0\right)=0$. We can suppose, without loss of generality, that $x_{0}=0$. Linearizing eq. (2.1) at 0 we obtain the system (6.10) with $A=D F(0,0), B=\frac{\partial F}{\partial \xi}(0,0)$.

Proposition 6.4. If $\Pi$ is $\delta$-narrow at $x_{0}=0$ with a sufficiently small $\delta$ then system (2.1) is stochastically stable only if

$$
\operatorname{dim} \operatorname{Span}\left\{B, A B, A^{2} B, \ldots, A^{n} B\right\}<n, \quad A=D F(0,0), B=\frac{\partial F}{\partial \xi}(0,0) .
$$

Proof. Consider any $x_{1}$ such that $\left|x_{1}\right|=2 \delta$ amd $x_{1} \notin \Pi$. According to the Kalman criterium, there exists a control $\xi(t), t \in\left[0, T_{0}\right]$ such that the corresponding trajectory of linear system (6.10) attains the point $\delta^{-1} x_{1}$. It is clear that $\xi(t)$ is independent of $\delta$, therefore $|\xi|<C$ where $C$ is uniform in $\delta$. Then the control $\delta \xi(t)$ gives a trajectory of $(6.10)$ that attains the point $x_{1}$.

Consider the same control $\delta \xi$ for the original system (2.1). For small $\delta$ by an estimate analogous to estimates from proof of Prop. 6.2, we can show that the corresponding trajectory of nonlinear system (2.1) leaves $\delta$-neighborhood of 0 . This proves our assertion.

This assertion shows that the analysis of stochastical stability of equilibria reduces to solution of the complicated system of polynomial equations:

$$
\begin{gathered}
\operatorname{dim} \operatorname{Span}\left\{B, A B, A^{2} B, \ldots, A^{n} B\right\}<n, \quad F(x, 0)=0 \\
A(x)=D F(x, 0), B(x)=\frac{\partial F}{\partial \xi}(x, 0) .
\end{gathered}
$$

In general, this system is overdetermined and one can expect that generically this system has no solutions and thus equilibrium states of (2.1) are stochastically unstable.

\section{$7 \quad$ Stability of evolving systems. General approach}

The main idea is based on the following observation: Lemma 6.1 of the previous section does not hold if system (2.2) contains internal parameters. In fact, let us consider (6.1) with $f, g$ 
and $h$ depending on some parameters $y \in \mathbf{R}^{n}$. "Generic" symmetric control systems defined by $g, h$ are not completely controllable since now for some $y$ the system

$$
g(x, y)=h(x, y)=0
$$

can, even in a generic case, have a solution $x$. Similarly, for polynomial dynamical systems (2.1) with $F=F(x, y, \xi)$ we seek for $y$ such that the system

$$
\begin{gathered}
\operatorname{dim} \operatorname{Span}\left\{B, A B, A^{2} B, \ldots, A^{n} B\right\}<n, \quad F(x, y, 0)=0, \\
A(x)=D F(x, 0), B(x)=\left.\frac{\partial F}{\partial \xi}(x, y, \xi)\right|_{\xi=0}
\end{gathered}
$$

is resolvable. We suppose that coefficients of polynomials involved in relations (7.2) lie in $h \mathbf{Z}$, where $h$ is a rational positive number.

This problem is well known in real algebraic geometry and named "elimination of quantifiers" (see [35]). Effective algorithms for this problem were found by D. Grigoriev et al. [36], another method was proposed by M. F. Roy et al. (see book [35]). The known algorithms take an exponential number $N_{E}$ of steps

$$
N_{E}=(d n)^{O\left(n^{2}\right)}
$$

where $d$ is the maximal degree of polynomials $A, B, F$ in $x$ and $y$. Notice that, in general, the problems of quantifier elimination or even of solvability of polynomial systems are NP-hard $[43]$.

Let us study now some mathematical models of internal parameter evolution. First we consider the case when our family of evolving systems consists of $N$ members defined by the parameters $y_{1}, \ldots, y_{M} \in \mathcal{M}$, where the set of possible values of the parameter $\mathcal{M}$ is finite or countable: $|\mathcal{M}|=\mathcal{M}$. We suppose that the evolution process is a Markov process with discrete time $t=0,1,2, \ldots$. The case of continuous time can be considered in an analogous way.

\subsection{Optimal structure of a Markov evolution for finite M}

Denote $p_{i}(t)$ the probability to be in the state $y_{i}$ at the moment $t$. Then the time evolution of $p_{i}(t)$ is subject to the equation

$$
p_{i}(t+1)=\sum_{j=1}^{M} w_{i j} p_{j}(t)
$$


where $w_{i j}$ is the transition probability from $y_{j}$ to $y_{i}$. To describe destruction of the states $y_{i}$ under a fixed external perturbation, we introduce an absorbing state 0 (the system is destroyed) and the corresponding probability transitions $q_{i}=w_{0 i}>0$ and $w_{i 0}=0$ (the last relations expresses the fact that we cannot return to $y_{i}$ from 0$)$. We assume that $q_{i}$ are small with respect to $\min \left\{w_{i j}>0\right\}$. Entries $v_{i j}$ are small pertubations of $w_{i j}$ such that

$$
\sum_{j=1}^{M} v_{j i}=q_{i}, \quad\left|v_{i j}\right|,\left|q_{i}\right|<\epsilon
$$

where $\epsilon>0$ is a small parameter. This means that the environment weakly influences, by $V$, on the coefficients $w_{i j}$ describing "an internal dynamics" of the system given by (7.4).

Then the complete evolution system consists of the modified eq. (7.4)

$$
p_{i}(t+1)=\sum_{j=1}^{M}\left(w_{i j}-v_{i j}\right) p_{j}(t)
$$

plus the equation

$$
p_{0}(t+1)=\sum_{j=1}^{M} q_{j} p_{j}(t)+p_{0}(t)
$$

determining a Markov evolution with $M+1$ states. System (7.4a), (7.6) can be asymptotically resolved for small $\epsilon$ by the standard methods. We denote by $\pi_{i}$ an equilibrium state of (7.4) defined as a positive solution of the eigenvalue problem

$$
\lambda \pi_{i}=\sum_{j=1}^{M} w_{i j} \pi_{j}
$$

with $\lambda=1$. Using the algebraic theory of Markov chains [28] and an elementary theory of perturbations, we obtain

$$
p_{i}(t)=C(p(0))\left(\lambda_{\epsilon}^{t} \beta+o(1)\right), \quad \epsilon \rightarrow 0,
$$

for large $t$, where the constant $C$ depends on the initial data and $\lambda_{\epsilon}=1-\beta, \quad \beta=\sum_{i=1}^{M} \pi_{i} q_{i}$. The correction $\beta$ describes a small change of the principal eigenvalue $\lambda=1$ of (7.7) under the small perturbation $V$. Substituting result (7.8) in (7.6), we have

$$
p_{0}(t)=C\left(1-\lambda_{\epsilon}^{t}\right)(1+o(1)), \quad \epsilon \rightarrow 0
$$


Given $q_{i}$, we seek an "optimal" process ( an optimal choice of $w_{i j}$ ) that minimizes $p_{0}(t)$ (the probability to be destroyed). It is easy to show that this optimum problem is independent of a concrete value $t$ for large $t>\epsilon^{-1}$ and reduces to a minimization of $\beta$. For this optimal process we have

$$
\sum_{i=1}^{N} \pi_{i} q_{i}=\min , \quad \sum_{i=1} \pi_{i}=1, \quad \pi_{i} \geq 0 .
$$

Suppose $q_{j}$ are different (that is a "generic" case). It is not difficult to see that the solution can be described as follows. Suppose $i_{0}$ is an index corresponding to the minimal $q_{i}$. Then we conclude that the optimal distribution $\pi_{i}$ is concentrated at $i_{0}: \pi_{i}=\delta_{i, i_{0}}$. This means that under a fixed random perturbation evolution must be selective, i.e., evolution seeks for a state $y_{j}$ that gives the maximal survival probability. These facts can be summarized by

Proposition 7.1. Under above assumptions, the process (7.4a)-(7.5) providing a maximal survival probability for each $T$ has the following structure: there is an index $i_{0}$ such that

$$
\pi_{i}=\delta_{i i_{0}}, \quad w_{i i_{0}}=\delta_{i i_{0}}
$$

The first relation means that the system is in an "optimal" state. The second relation means that the process makes copies of an "optimal" state.

Let us consider now a more complicated case, namely, when there are possible different perturbations leading to destruction of states and it impossible to foresee which from them acts. This means that the random environment, in a sense, is nonpredictable.

To describe this situation, we use the following notation. Let $\omega \in \Omega_{0}$ be a random parameter that defines a choice of a random external perturbation. The quantities $q_{i}$ depend on $\omega$ : $q_{i}=q_{i}(\omega)$. The optimal structure of the process can be found now as a solution of the following minimax problem: to find $\pi_{i}$ minimizing

$$
\max _{\omega \in \Omega_{0}} \sum_{i=1}^{M} \pi_{i} q_{i}(\omega), \quad \sum_{i=1} \pi_{i}=1, \quad \pi_{i} \geq 0 .
$$

In this case, in general, equilibrium state probabilities $\pi_{i}$ are localized at some different points. We conclude thus that in a non-predictable random enviroment the evolution must be more random than in a predictable one.

Let us show that genetic evolution actually posesses similar properties. We remind the classical model proposed by R. Fisher [38, 39]. Assume the genetic structure of the population 
consists of $m$ alleles $y_{j}$ (different variations of the same gene). The population size is $X(t)$. The probability that a member of the population has the $j$-th allel, is $p_{j}$. According to $\mathrm{R}$. Fisher, time evolution of these allel frequencies is defined by the equations

$$
\frac{d p_{i}}{d t}=\frac{\partial W(p)}{\partial p_{i}}+\kappa(X, p) \zeta_{i}(t)
$$

where $p=\left(p_{1}, p_{2}, \ldots, p_{m}\right)$ is the vector of the allel frequencies, $W$ is the so-called fitness of the population, $\zeta_{i}$ are white noises and $\kappa \approx X^{-1 / 2}$.

The term with $\xi_{i}$ describes random contributions to gene dynamics ( the genetic drift). We add to (7.11) the classical equation for the population dynamics:

$$
\frac{d X}{d t}=r X(K-X)
$$

where $r$ is a coefficient determining the rate of the population growth, $K$ is the so-called capacity, i.e., a maximal population size that can be attained in a given ecological environment (due to a simple fact that resources are restricted). Coefficients $r, K$ can depend on $p_{i}$.

In a stable environment, without any ecological catastrophes, the population size $X$ is large enough and the terms with $\kappa$ are small. If we set $\kappa=0$, i.e. we remove the noises, we observe that the complete population fitness $W(p)$ grows with time $t: d W / d t>0$ on the trajectories (7.11) (Fisher's theorem, [38, 39]). This means that the complete fitness increases during evolution. The term with the gradient of $W$ describes a natural selection, when evolution seeks for $p_{i}$ with the maximal fitness $W(p)$.

Let us consider an ecological catastrophe. Here we dealing with unpredictable perturbations (see above, eq. (7.10)), since the population, at the moment of this catastrophe, cannot foresee the type of environment after catastrophe. Thus evolution must be random.

Let us show now that, in this situation, system (7.11), (7.12) proceeds a more random evolution. We can suppose that an ecological catastrophe leads to a strong sudden fall of the value of $K$. Then $X$ falls as well and therefore the terms $\sigma \xi_{i}$ could become much more essential than $\nabla W$, i.e., equations (7.11) describe a random search.

To conclude this subsection, let us notice that if all $q_{i}>0$, then for a fixed $M$ the probability $P_{T}$ to survive during time interval $[0, T]$ converges to zero as $T \rightarrow \infty$. (It follows from relation $(7.8))$.

Therefore, to survive we should increase $M$ from time to time introducing new states. In this case we obtain a Markov chain with an infinite number of states plus a marked state 0 . 
Then it is well known that the probability to be absorbed by 0 may be $<1$ [28]. This means a possibility of a stable evolution for large times. To describe a more complicated situation with $M=\infty$, we consider a particular case: branching processes.

\subsection{Evolution for countable state sets: branching processes, algorithms, NP- hardness and evolution properties}

We suppose here, that, at each time moment $t$, the state $y$ may proceed to new states

$y_{1}^{\prime}, \ldots, y_{n(t, y)}^{\prime}$. The number $n$ of new potentially possible states is finite but it may depend on the moment of time and the previous states. We can imagine an evolution "tree" growing in time, where, at each node, we can go to any branch describing a new state. During this motion, a perturbation can destroy old states.

We state the following problem: how to estimate the size of the evolution tree providing a stable evolution, when the survival probability limit $P_{T}$ stays greater than a positive constant? (i.e., the limit relation $P_{T} \rightarrow 0$ as $T \rightarrow \infty$ does not hold). Our goal is to explain increasing of evolution tree and genetic code with time growing. The main idea is to connect this problem with the theory of algorithmic complexity. To create a system making a stable homeostasis, is a complex problem (see above, Sections 5, 6).

To formalize more the problem and to apply to it the theory of algorithms, we assume the following. Let us suppose that each state $y$ is defined by a code $C_{y}$. To simplify, we consider the problem with discrete time: $t=0, \tau, 2 \tau, \ldots$, where $\tau$ is a time step. At each instant of time, we transform this code to another code.

We suppose as well that the survival problem has some "a priori computational complexity" $C o m p_{a}$. Let us observe that there exists a tradeoff between a memory $\mathrm{Mem}$ needed to perform an algorithm and the number of steps $N_{\text {time }}$ of this algorithm. For certain problems there was obtained the estimate (first it was obtained in [41], see book [37] for a review):

$$
\mathrm{Mem} \cdot N_{\text {time }} \geq \text { Comp }_{a}
$$

We can illustrate this fundamental relation by an example, namely, by the famous salesman problem. Let us consider $n$ cities located in a country. Distances between cities are given. The problem is to find a tour running all $n$ cities (each city once) and having the minimal length. Here the algorithm of the exaustive search has an exponential time cost $N_{\text {time }}=O(n e)^{n}$ but it uses the memory $O(n)$. On the other hand, if we use a memory $2^{n}$, we can solve the salesman 
problem in $O(n)$ steps (see [44]).

Remark: It is important to note that if $P \neq N P$ and, for a NP-complete problem the $N_{\text {time }}$ depends polynomially on the input size $|C|$ (the code size), and Compapriori is not polynomial in $|C|$, then the memory size should be non-polynomial in $|C|$.

Furthermore, we suppose that the evolution solves a chain of computational problems to survive. Namely, we deal with problems $P r_{1}, \ldots P r_{k}, \ldots$ of increasing a priori complexities $\operatorname{Comp}_{a}(1), \operatorname{Comp}_{a}(2), \ldots, \operatorname{Comp}_{a}(k), \ldots$ Each solution of each problem is defined by some codes corresponding to our states.

Let us formulate an important assumption.

Assumption 7.1 At the moment $t$, all states with the code $C_{y}$ can be destroyed simultaneously by the random environment within time interval $[t, t+1]$ with the probability $Q(y)$ independent of $t$ (thus we suppose that the random processes are homogeneous in time).

Example. Let us turn to the model from Problem 5.1 in Section 5. Recall that given nodes $s_{i}$ could correspond to nutrients (substrates), given nodes $\bar{s}_{i}$ could correspond to products, the unknown paths correspond then to some metabolic paths. For this model the problem $\operatorname{Pr}_{k}$ is to find $k$ mutually disjoint paths from $s_{i}$ to $\bar{s}_{i}$, i.e., from subtrats to products. Under assumptions from Section 5, one has $Q(y)=r_{1} r_{2} \ldots r_{k}$.

We suppose, moreover, that if the corresponding code $C_{y}$ is a solution of the problem $\operatorname{Pr}_{k}$, then the probability $Q(y)$ satisfies

$$
Q(y)>1-f(k)>0
$$

where $f(k)>0$ is a function of the integer argument $k$. This means that, at each step, there is a uniform low bound for the destruction probability (depending on the step number).

By solving the chain of the computational problems the population increases the survival probability. The chances to survive depend on the evolution algorithm speed and on the environment properties.

Let us introduce the quantity $S_{e v}(k)$, which is the number of the states (the nodes of the evolution tree ) with pairwise different codes obtained to this moment, when $k$-th computational problem is resolved. Notice that only different codes are essential for evolution, it follows from Assumption 7.1. Moreover, let us observe the inequality $S_{e v}(k) \leq \max |C(y)|$, where the maximum is taken over all states at the $k$-th step. Therefore, if the tree is large, the code length also is large. 
Proposition 7.2 Suppose that the evolution is stable, i.e., $P_{T} \rightarrow p_{\infty}>0$ as $T \rightarrow \infty$. Assume that the evolution solves a sequence of computational problems (as described above) such that their a priori complexities $\mathrm{Comp}_{a}(k)$ increase faster in $k$ than any polynomial $k^{O(1)}$ and that for these problems the estimate (7.13) holds. Assume that the population is in an hard environment, i.e., relation (5.5) holds

$$
f(k)<1-\delta k^{-\mu}, \quad \delta, \mu>0 .
$$

Then, if $P \neq N P$, the code size $|C(y)|$ and the evolution tree size $S_{\text {ev }}(k)$ tend to $\infty$ as $k \rightarrow \infty$.

Proof. Suppose that $|C(y)|$ are bounded for all $k$. Then the quantities $S_{e v}(k)$ are bounded as well (see above). Our plan is to find an estimate of the running time $T_{\text {run }}(k)=\tau_{k}$ at $k$-th step of evolution, when $k-1$ problems have been resolved. Due to Assumption 1, the probability $q_{k}$ that all members of the population with $S_{e v}(k)$ members will be destroyed within the time interval $\tau_{k}$ can be computed in an elementary way. We observe that the probability of survival of a population member is $1-(1-Q(y))_{k}^{\tau}$. Thus

$$
q_{k}=\left(1-\left(1-(1-Q(y))_{k}^{\tau}\right)^{S_{e v}(k)}\right.
$$

and, by inequality (7.14), this quantity satisfies

$$
q_{k}>\left(1-f(k)^{\tau_{k}}\right)^{S_{e v}(k)} .
$$

Thus the probability $p_{k}$ that at least one member of the population survives satisfies

$$
p_{\infty}<p_{k}<1-\left(1-f(k)^{\tau_{k}}\right)^{S_{e v}(k)} .
$$

This gives

$$
\alpha=-\log \left(1-p_{\infty}\right)<S_{e v}(k)\left(-\log \left(1-f(k)^{\tau_{k}}\right)\right)
$$

and

$$
S_{e v}\left(k_{1}\right)>\frac{\alpha}{\left(-\log \left(1-f\left(k_{1}\right)^{\tau_{k_{1}}}\right)\right)}
$$

for any $k_{1} \in\{1,2, \ldots, k\}$. Let us take a sufficiently large $\gamma$ such that $\gamma>\mu+2$, where $\mu$ is taken from (7.15). For times $\tau_{1}, \tau_{2}, \ldots$ we have (if $P \neq N P$ and (7.13) holds)

$$
\tau_{1}+\tau_{2}+\ldots+\tau_{k} \geq c_{1} k^{\gamma_{1}}, \quad \gamma_{1}=\gamma-1,
$$


since the sum in the left hand side is the complete running time for $k$-th problem with memory $O(k)$. Therefore, there exists an integer $k_{*}, 0<k_{*} \leq k$ such that

$$
\tau_{k_{*}}>c_{2} k^{\gamma_{1}-1}
$$

Let us substitute this $k_{*}$ in inequality (7.16). We observe then that $f_{k_{*}}^{\tau_{k_{*}}}<1 / 2$ for $k$ large enough and, since $-\log (1-x) \leq 2 x$ for $x \in(0,1 / 2)$, this implies

$$
S_{e v}\left(k_{*}\right)>\frac{\alpha}{2} f\left(k_{*}\right)^{-\tau_{k_{*}}}=h\left(k_{*}\right) .
$$

From (7.15) it follows that $f\left(k_{*}\right)<1-\delta k_{*}{ }^{-\mu} \leq 1-\delta k^{-\mu}$. Therefore,

$$
h\left(k_{*}\right)>\frac{\alpha}{2}\left(1-\delta k^{-\mu}\right)^{-\tau_{k_{*}}} .
$$

Using (7.17) we notice that $h \rightarrow \infty$ as $k \rightarrow \infty$. We have obtained a contradiction with our hypothesis that $|C(y)|$ (and thus $S_{e v}(k)$ ) are uniformly bounded in $k$. The proposition is proved.

Remark 1. The main idea is as follows: an algorithm resolving a complicated evolution problem uses a tradeoff between running time and memory, see (7.13), since the time to create a stable structure is restricted.

Remark 2. Many NP-hard problems such as k-SAT [43] or certain graph problems can be resolved, under some conditions, for "almost all" instances, by effective greedy algorithms. In this case the evolution problems can be resolved fast and without big trees since then $\tau_{k}<$ const.

Consider an example. Let us look at Problem 5.1. To find a simple path from node $s_{1}$ to node $t_{1}$, we can use, for example, the Dijkstra algorithm, which is greedy [40]. The running time is $O(|V| \log |V|)$ for graphs with a bounded averaged valency. If we have $k-1$ simple ways from $s_{i}$ to $t_{i}, i=1, \ldots k-1$, we can remove edges and nodes involved in these ways and again seek for a way from $s_{k}$ to $t_{k}$. Then $\tau_{k}=O(|V| \log |V|)$ and it is independent of $k$. It is clear, however, that such an approach does not work for arbitrary graphs, since this way could be absent. However, one can expect this approach to be successful for almost all graphs (with respect to an appropriate measure) with sufficiently large averaged valency.

\section{Conclusion: biological interpretations of results and connections with experiments}

Results on circuit model (2.7) are consistent with experimental data. First we summarize our results for the gene circuits. We have obtained that 
C1. The sharper is the sigmoidal function $\sigma$, the larger is the survival probability; it is consistent with general ideas on enzyme dynamics [19].

C2. The more is the valency of a node the stabler is the circuit with respect to perturbations in this node. This conclusion is in an accordance with experimental results of the work [30]. They show that the most connected proteins in the cell are the most important for its survival.

C3. Investigating evolution process we have found that the averaged valency should increase. This conclusion also is confirmed by experimental data (see [29]). Notice as well that for graphs with a larger averaged valency it is simpler (with a larger probability to find a solution) to resolve NP-hard problems having biological meaning and mentioned in Section 5.2.

Moreover, it is shown (see Example 2, Section) that the preferential attachment evolution algorithm is stabler than the Erdos -Rényi algorithm. This means that the principle of stochastic stability in a random environment explains why biological networks have free-scale structure and why Erdos-Rényi structure is unstable. Evolution algorithms have to use variants of preferential attachment.

Let us state now a summary of the results for more general systems (2.1), (2.2). We show that a "generic system" is unstable under random perturbations. This property leads to important consequences.

G1. The averaged time of species existence may be large but it is bounded. The species living in a stabler environment should have, in average, a larger existence time than the species living in an unstable environment.

G2. If our random environment is, in a sense, predictable, i.e., this environment generates a stationary noise with parameters that rest the same during a very large time period, then an optimal evolution algorithm should be more "selective", i.e., this algorithm should seek for an optimal genetic structure.

In opposite, if the environment is non-predictable, i.e., there are possible ecological catastrophes that sharply change the environment from time to time, then the evolution algorithm should be more randomized.

The next conclusion follows from the complexity theory of algorithms (section 7).

G3. If the evolution solves a problem that can be interpreted as a hard computational problem, then the evolution tree must be very large: evolution, to be stable under pertur- 
bations, should produce many different new states. It is clear as well that the evolution, in a medium with restricted resources, should use the death in order to be successful: old copies must be eliminated.

One can assume that, at initial studies of evolution, primitive beings living in a hard environment have used this strategy of fast reproduction to survive (so-called $r$-strategy, see [7]). Our hypothesis is that the evolution has invented effective greedy algorithms corresponding $K$ -strategy (when replication produces a small number of well adapted beings, [7]).

To conclude, let us notice that, of course, the models studied here are strongly simplified. For example, we have assumed that the environment does not depend on the population state, i.e., there is no feedback between evolving systems and environment. Really, it is clear that this assumption is not quite correct and can be considered only as a first approximation.

\section{Ackowledgements}

The authors are thankful to Dr. S. Genieys and Dr. J. Reinitz for useful remarks. We are very grateful to Dr. O. Radulescu for interesting discussions. The first author was supported by the grant PAST ( University Lyon 1, France) and by the grant CNRS (University Rennes 1).

\section{References}

[1] R. Thom, Stabilité structurelle et morphogénèse, New York, Benjamin, 1972.

[2] R. Gilmore, Catastrophe Theory for scientists and engineers, John Wiley and Sons, New -York etc. 1981.

[3] Dynamical Systems with Hyperbolic Behaviour, D. V. Anosov (ed). (Dynamical Systems 9). Encyclopedia of Mathematical Sciences v. 66). Translated from Russian., Springer V., Berlin, Heidelberg, New-York, 1995.

[4] Smale S., Mathematics of Time, Springer, New - York, 1980.

[5] Ruelle D., Elements of differentiable dynamics and bifurcation theory, Acad. Press, Boston, 1989. 
[6] T. Brocker, K.Lander, Diffential Germs and Catastrophes, Cambridge Uiniversity Press, 1975.

[7] M. Begon, J.L. Harper, C. R. Townsend, Ecology, V2, Blackwall Scientific Punlications, Oxford London Edinburgh, 1986.

[8] R. May, Will a large complex system be stable, Nature(London), 238, 413-414, 1972.

[9] C. Lobry, Une propriete generique des couples de champs de vecteurs, Chechoslovak Mathematical Journal, 22 (97), p. 230-237, 1972.

[10] M. M. Hirsch, Differential topology, Springer-Verlag, New-York Heidelberg Berlin 1976.

[11] Yu. Ilyashenko, Weigu Li, Nonlocal bifurcations, AMS, 1999.

[12] P. Erdos, A. Rényi, On the evolution of random graphs, Publ. Math. Inst. Hungarian Academy of Sciences 5, p.17-61, 1960.

[13] Kolchin V. F., Random graphs. FizMatLit. 2004.

[14] R. Albert and A. L. Barabási, Statistical mechanics of complex networks, Rev. Modern Physics, 74, (2002) 47-97

[15] W. Horsthemke, R. Lefever, Noise-induced Transitions, Springer-Verlag, Berlin etc. 1984.

[16] J. J. Hopfield, Neural networks and physical systems with emergent collective computational abilities, Proc. of Natl. Acad. USA, 79 (1982) 2554-2558.

[17] B. Alberts, D. Bray, J. Lewis, M. Raff, K. Roberts, P. Walter, Molecular Biology of the Cell, 4nd ed. (Garland Publishing, Inc., New York, 2002)

[18] M. Ridley, Evolution, 2nd ed. (Blokwell Scientific Publications Ltd, Oxford, 1996)

[19] A. L. Lehninger, D. L. Nelson and M. M. Cox, Principles of Biochemistry, 2nd. ed. (Worth, New York, 1993).

[20] L. Glass and S. Kauffman, The logical analysis of continuous, nonlinear biochemical control networks, J. Theor. Biology, 34, (1973), 103-129. 
[21] D. Thieffry and R. Thomas, Dynamical behaviour of biological regulatory networks, II.Immunity control in bacteriophage lambda, Bull. Math. Biology, 57, (1995) 277-295.

[22] R. Edwards, T.H. Siegelmann, K.Aziza and L. Glass, Symbolic dynamics and computation in model gene networks, Chaos, 11 , (2001) 160-169.

[23] P. Smolen, D. Baxter, J. H. Byrne, Mathematical modelling of gene networks, Review in Neuron, 25, (2000) 247-292.

[24] E. Mjolness,D. H. Sharp and J. Reinitz, A connectionist Model of Development, J. Theor. Biol. 152, (1991) 429-453.

[25] J. Reinitz and D. H. Sharp, Mechanism of formation of eve stripes Mechanisms of Development, 49, (1995) 133-158.

[26] I. Salazar-Ciudad, J. Garcia-Fernadez and R.V. Solé, Gene Networks Capable of Pattern Formation: From Induction to Reaction-Diffusion, J. Theor Biology, 205 (2000), 587-603.

[27] L. Mendoza and E. R. Alvarez-Buylla, Dynamics of Genetic Regulatory Networks for Arabodopsis thaliana Flower Morphogenesis, J. Theor. Biol. (1998), 193, 307-319.

[28] S. Karlin, A first course in stochastic processes, Academic Press, New York London 1968.

[29] H. Jeong, B. Tombor, R. Albert, Z. N. Otvai and A.L. Barabási, The large-scale organisation of metabolic networks, Nature (London), 407, (2000) 651-654.

[30] H. Jeong, S. P. Mason, A.L. Barabási and Z. N. Otvai, Lethality and centrality in protein networks, Nature (London), 411, (2000) 41-42.

[31] L. H. Hartwell, J. J. Hopfield, S. Leibler and A. W. Murray, From molecular to modular cell biology, Nature(London), 402, (1999) C47-C52.

[32] M. Gromov and A. Carbone, Mathematical slices of molecular biology, Preprint IHES/M/01/03, 2001.

[33] A. D. Ventsel and M. I. Freidlin, Random Perturbations of Dynamic Systems (Springer, New York, 1984). 
[34] I. I. Gichman, A. B. Scorochod, Introduction to Theory of Random Processes (in Russian, Nauka, Moscow, 1977).

[35] S. Basu, R. Pollack, M. F. Roy, Algorithms in real algebraic geometry, Berlin-Heidelberg, New-York, Springer, 2003.

[36] D. Grigoriev, Complexity of deciding Tarski algebra, J. Sym. Computations, 1988, Vol. 5, 65-108.

[37] J. E. Savage, Models of Computations. Exploring the Power of Computing, AddisonWesley, 1997.

[38] Fisher, R. A.: The genetical theory of natural selection. Oxford, Clarendon Press, 1930.

[39] Sviregev, Ju. M. and Pasekov V. P.: Foundations of theoretical genetics (Moskow: Nauka) 1982

[40] T. H. Cormen, C. E. Leiserson, R. L. Rivest, Introduction to Algorithms, MIT Press, New York etc. 1990.

[41] D. Grigoriev, Application of separability and independence notions for proving lower bounds of circuit complexity, J.Soviet Math., vol. 14, 5, pp.1450-1456, 1980.

[42] J. D. Murray, Mathematical Biology, (Springer, New York, Berlin, Heidelberg, 1993).

[43] M. H. Garey, D. S. Jonnson, Computers and Intractability, A Guide to the Theory of NP-completeness, W. H. Freeman and Company. New York, 1979.

[44] C. H. Papadimitriou, K. Steglitz, Combinatorial optimization, Algorithms and Complexity, Prentice Hall, Inc. Englewood Cliffs. Ne-Jersey, 1982

[45] S. Vakulenko, D. Grigoriev, Complexity of gene circuits, Pfaffian functions and the morphogenesis problem, C. R. Acad. Sci, Ser I. 337 (2003) 721-724 